\title{
The Higher Number and Longer Duration of Kenogen Hairs Are the Main Cause of the Hair Rarefaction in Androgenetic Alopecia
}

\author{
Marcella Guarrera Alfredo Rebora \\ Department of Dermatology, DISSAL, University of Genoa, Genoa, Italy
}

\section{Keywords}

Androgenetic alopecia - Baldness · Kenogen · Finasteride · Hair cycle

\begin{abstract}
The progressive reduction of the number of terminal hairs and the simultaneous increase in the number of vellus hairs (hair miniaturization) are the current explanation of human baldness. In a 2.5-year study on 43 male subjects with androgenetic alopecia (AGA), we found that oral finasteride augmented the total number of hairs by $55 \%$, but failed to decrease the prevalence of vellus hairs. While our data may explain why, in AGA, hairs become progressively softer and combable, they cannot explain why areas of hair rarefaction develop. We propose that the longer duration and higher frequency of kenogen hairs are the real mechanism through which the scalp hairs rarefy.

(C) 2018 S. Karger AG, Basel
\end{abstract}

\section{Introduction}

In both genders, androgenetic alopecia (AGA) is characterized not only by the progressive reduction of the number of terminal hairs, but also and especially by the simultaneous increase in the number of vellus hairs, which are currently regarded as the markers of the disorder. This event, named hair miniaturization, is considered the main pathogenetic factor of AGA.

In a large multicenter study, finasteride proved many years ago to induce a partial reversal of AGA [1]. Much data has been produced to support such beneficial effect, including the increase in the weight of hair fibers [2], which, given the genetic fixity of the number of hair follicles, was interpreted as the result of the reversal of vellus hairs to the bigger terminal hairs. Although such interpretation is the one clinicians use to offer to their balding patients, it hardly explains why areas of diminished hair density become progressively visible on their scalp.

In the present study, oral finasteride confirmed its efficacy in increasing the total number of hairs, but failed to decrease the prevalence of vellus hairs. In the light of the mentioned interpretation, this may explain why, in AGA, hairs become progressively softer and combable, but cannot explain why areas of hair rarefaction develop. A novel interpretation seems necessary.

\section{Subjects and Method}

Forty-three males with AGA in different Hamilton's stages (II to IV) (mean $2.8 \pm 0.7$ ), aged $21-45$ years (mean $29.4 \pm 6.1$ ), were studied. The study was approved by the Institute's Committee and all subjects signed informed consent. After a 6-month washout (c) 2018 S. Karger AG, Basel

E-Mail karger@karger.com

www.karger.com/sad
Alfredo Rebora, MD

DISSAL

Via Pastore 1

IT-16132 Genoa (Italy)

E-Mail rebdermo@yahoo.it 


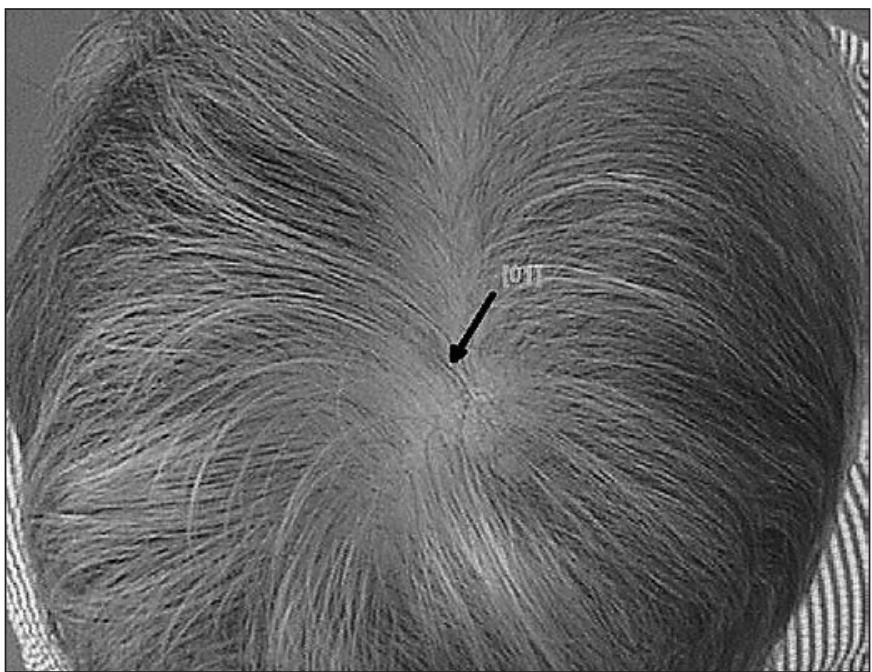

Fig. 1. The mark of the videodermoscope on the vertex.

Table 1. Average number of terminal and vellus hairs

\begin{tabular}{|c|c|c|c|c|c|c|}
\hline \multirow[t]{3}{*}{ Steps } & \multirow{3}{*}{$\begin{array}{l}\text { Subjects, } \\
n\end{array}$} & \multicolumn{5}{|l|}{ Hairs } \\
\hline & & \multicolumn{2}{|c|}{ terminal } & \multicolumn{3}{|l|}{ vellus } \\
\hline & & $n / \mathrm{cm}^{2}$ & $\pm \mathrm{SD}$ & $\mathrm{n} / \mathrm{cm}^{2}$ & $\%$ & $\pm \mathrm{SD}$ \\
\hline T0 & 43 & 170.1 & 50.2 & 16.5 & 9.7 & 5.0 \\
\hline T3 & 25 & 180.1 & 53.7 & 15.5 & 8.6 & 6.2 \\
\hline T6 & 20 & 183.8 & 63.8 & 15.2 & 8.3 & 6.2 \\
\hline $\mathrm{T} 12$ & 14 & 162.9 & 46.3 & 16.6 & 7.9 & 4.0 \\
\hline T18 & 9 & 200.1 & 47.2 & 19.0 & 9.5 & 4.8 \\
\hline $\mathrm{T} 24$ & 4 & 187.8 & 30.0 & 16.9 & 9.0 & 1.6 \\
\hline T30 & 2 & 263.8 & 26.6 & 25.4 & 9.6 & 2.1 \\
\hline
\end{tabular}

period from any hair loss treatment, they were given finasteride (1 $\mathrm{mg} /$ day) for 30 months and planned to be examined after 3 (T3), 6 (T6), 12 (T12), 18 (T18), 24 (T24), and 30 (T30) months. On all occasions, the subjects were submitted to a videodermoscope provided with a software (FotoFinder, Trichoscan software, Bad Birnbach, Germany). They were photographed globally and, at 20x magnification, in a small area of the scalp vertex of $0.651 \mathrm{~mm}^{2}$, identified by the machine itself (Fig. 1), after clipping the hair shafts at 1-2 mm length. The hair density (number $/ \mathrm{cm}^{2}$ ) and the percentage of vellus were measured. Data were submitted to the $t$ test, and $p<0.05$ was considered statistically significant.

\section{Results}

The main results are shown in Table 1. Fourteen subjects dropped out, and only two completed the full course. The remaining 29 subjects missed one or more steps.
Density increased from $170.1 \pm 50.2$ at $\mathrm{T} 0$, to $180.1 \pm 53.7$ at $\mathrm{T} 3$, to $183.8 \pm 63.8$ at $\mathrm{T} 6$, to $162.9 \pm 46.3$ at $\mathrm{T} 12$, to 200.1 \pm 47.2 at $\mathrm{T} 18$, to $187.8 \pm 30.0$ at $\mathrm{T} 24$, to $263.8 \pm 26.6$ at T30. At the end of the study, the number of terminal hairs had increased by $55 \%$. The difference was statistically significant $(t=2.622 ; p=0.013)$.

Vellus hairs remained stable, though with minor fluctuations, through the 2.5 years of the study, averaging $8.9 \%$ of all hairs. The difference was not statistically significant $(t=0.028 ; p=0.978)$. The absolute count of vellus hairs was stable as well, except for a single final increase.

\section{Discussion}

In our study, the increase in the number of terminal hairs induced by finasteride was not accompanied by the decrease in the percentage of vellus hairs as it should have done if the pathogenetic mechanism of progressive miniaturization were the real mechanism producing hair rarefaction. A similar result had been obtained by Rushton et al. [3] and largely commented by Sinclair [4].

Limitations of our work were essentially those related to the large number of dropouts. We did not investigate the single reasons of that phenomenon, but considering that our study was exceedingly long and was not sponsored, and the subjects had no incentives whatsoever, it was to be expected. Plausibly, only subjects who were encouraged by the clinical success remained in the study. This interpretation, however, proved wrong by some subjects who did not come at one step but reappeared at the following.

Two possible explanations of the observed phenomenon can be conjectured. First, while transforming vellus hairs into terminal hairs, finasteride would be unable to prevent the miniaturization of further terminal hairs. Second, finasteride would succeed in awakening quiescent hairs (kenogen) [5], but failed to prevent further miniaturization.

If the first hypothesis were true, finasteride would be useless to the patient, for it would not result in an improvement of the disorder, which instead it does generate. The increase of terminal hairs would be, in fact, nullified by the simultaneous miniaturization of new hairs. The second hypothesis, instead, seems to us more likely.

Kenogen is a term used to indicate periods in which the follicle is free from the hair shaft. Those periods coincide with the interval between the telogen shedding and the emergence of a new anagen hair. Kenogen has been shown to occur in a prepubertal boy, in whom the main causal factors of AGA, namely testosterone and $5 a$-reductase, are deficient and absent, respectively, sug- 
2

Fig. 2. A normal scalp. Vellus hairs are very few and all terminal hairs surface by triplets or even quadruplets per each hair canal.

Fig. 3. Androgenetic alopecia. Vellus hairs are numerous, triplets are absent, and pairs are few. Numerous empty spaces are visible.

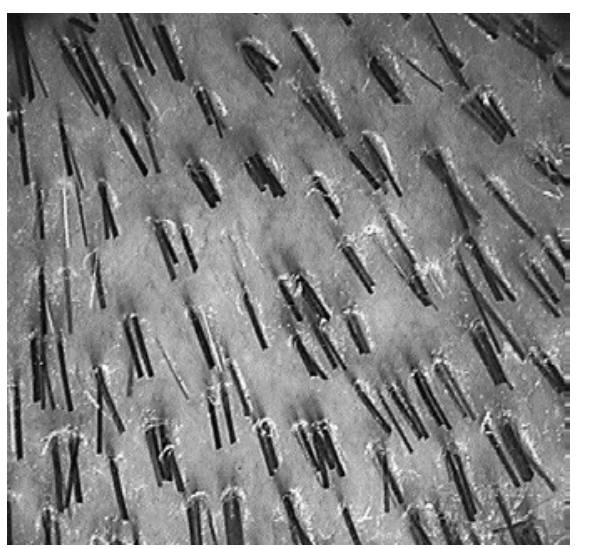

3

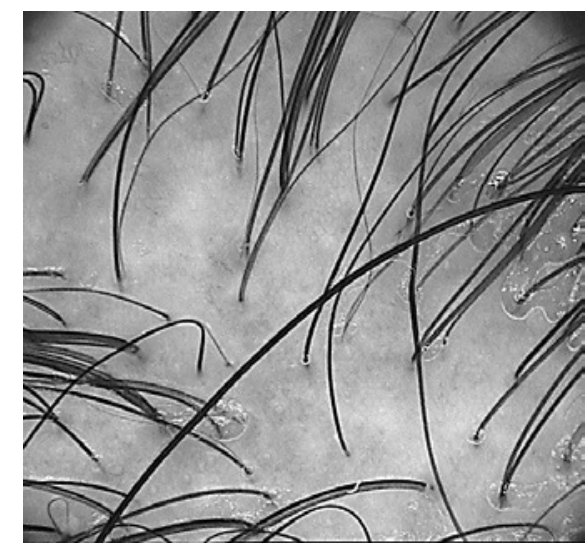

gesting that the phenomenon is a physiological one, possibly reflecting a period of true rest of the hair follicle [6].

In two women with progressing AGA studied for 2 years, kenogen has been shown to involve $22 \%$ of the hair follicles, lasting a variable time in the same subject (from 3 months to 1 year) [7]. Both frequency and duration were greater in the patient in whom AGA was more severe. The prevalence of kenogen hairs increased in parallel with the growing number of vellus hairs and the diminished number of normal hair cycles, features that both mark the AGA aggravation. In balding males as well, kenogen was observed to last 4-7 months and to affect $80 \%$ of all hair cycles [8].

Our findings suggest that the old concept of hair miniaturization as the sole possible cause of baldness is hardly tenable. In fact, baldness is an ambiguous term that includes two distinct phenomena: the progressive hair softness ladies complain of when they have their hair done, and the areas of hair rarefaction. If hair miniaturization explains the first feature, the kenogen hypothesis seems more likely to explain the second. The increased duration and frequency of kenogen are the real mechanism through which the scalp hairs rarefy.
As it is well known, hairs surface normally by triplets or even quadruplets per each hair canal (Fig. 2). We propose that, in turn, one hair per each follicular unit goes physiologically "on vacation" (kenogen). Physiologically, the "vacation" affects about $10 \%$ of all hairs and lasts about 2 months [6]. Over time, with progressing AGA, the number of hairs that go "on vacation" increases, affecting two and eventually even all hairs per follicular unit and the "vacation" lasts much longer, probably even years, causing a small area of hair rarefaction to develop (Fig. 3). Ultimately, the confluence of such areas produces the human baldness.

\section{Acknowledgment}

We thank Manuela Baldari, MD and Martina Montinari, MD for their invaluable help.

\section{Disclosure Statement}

The authors declare no conflict of interest.

\section{References}

1 Van Neste D, Fuh V, Sanchez-Pedreno P, Lopez-Bran E, Wolff H, Whiting D, et al. Finasteride increases anagen hair in men with androgenetic alopecia. Br J Dermatol. 2000 Oct; 143(4):804-10.

2 Price VH, Menefee E, Sanchez M, Kaufman $\mathrm{KD}$. Changes in hair weight in men with androgenetic alopecia after treatment with finasteride ( $1 \mathrm{mg}$ daily): three- and 4-year results. J Am Acad Dermatol. 2006 Jul;55(1): 71-4.
3 Rushton DH, Norris MJ, Van Neste D. Hair regrowth in male and female pattern hair loss does not involve the conversion of vellus hair to terminal hair. Exp Dermatol. 2016 Jun; 25(6):482-4.

4 Sinclair R. Androgenetic alopecia. Modelling progression and regrowth. Exp Dermatol. 2016 Jun;25(6):424-5.

5 Rebora A, Guarrera M. Kenogen. A new phase of the hair cycle? Dermatology. 2002; 205(2):108-10.
6 Guarrera M, Cipriani C, Rebora A. Delayed telogen replacement in a boy's scalp. Dermatology. 1998;197(4):335-7.

7 Guarrera M, Rebora A. Kenogen in female androgenetic alopecia. A longitudinal study. Dermatology. 2005;210(1):18-20.

8 Courtois M, Loussouarn G, Hourseau C, Grollier JF. Hair cycle and alopecia. Skin Pharmacol. 1994;7(1-2):84-9. 